\title{
Guidelines and Recommendations for the Development of Cross-Linguistic Awareness for Foreign Language Learning and Teaching
}

\author{
Tina Čok \\ Faculty of Humanities, University of Primorska, Slovenia \\ tina.cok@fhs.upr.si
}

\begin{abstract}
This paper aims to introduce new guidelines and recommendations for foreign language learning and teaching that have been conceived on the basis of data collected with an empirical research on discrepancies between conceptual representations of reality and their verbalisations by native speakers of Chinese, Italian and Slovenian. By analysing the results of the empirical research and comparing them to the currently established reference frameworks for learning and teaching foreign languages, we designed novel guidelines and recommendations that give special emphasis to the role of cross-linguistic competences in the process of foreign language learning and teaching and suggest a more comprehensive language reference framework that must consider and include these competences. The proposed guidelines and recommendations were designed to give support to learners and teachers in the process of learning and teaching Chinese as a foreign language. However, they can be easily adapted and applied for learning and teaching other foreign languages.
\end{abstract}

Keywords: cross-linguistic awareness, foreign language learning and teaching, verbal aspect

\section{Introduction}

The pedagogical framework embodied by guidelines and recommendations presented in the following pages is primarily based on empirical data acquired within a broader research study on verb conceptualisation and verbalisation in three different languages; Chinese, Italian and Slovenian.

The novelty of our research lays in the attempt of breaking away from the dominant approaches in studying semantic language differences that are usually based on notions of intercultural studies that explain linguistic differences in the light of different cultural patterns and contexts, which are consequently identified also in the language in question. Our comparison of similar communication situations in languages that differ morphosyntactically (i.e. Chinese, Italian and Slovenian) has made it possible to 
identify differences in comprehension of word meanings or entire messages through cross-linguistic notions analysed with the approaches of cognitive linguistics. Hence, the research principally aimed at exploiting the identified discrepancies in the conceptualisation and verbalisation of reality for three languages to improve foreign language learning in teaching.

We see the value of this research in addressing concrete communication problems that may arise in different situations. The results of this study can be applied for a more general understanding of Chinese social phenomena, often inconceivable or even unacceptable for people not native to $\mathrm{Chi}$ na. The principal idea of the present research is to stress the importance of comprehending the behaviour of a group of people who share similar mental processing and consequently contemplate the world in a similar way. It is said that the Chinese conception of the world is based on a holistic worldview, how people engage in relations and how they interact. This specificity of the Chinese society that complies with the notions of synthetic thinking, which makes it different from western thought, has given rise to discussions among psychologists, anthropologists, philosophers and cognitive linguists for decades. Our share in this debate is to empirically analyse this worldview by considering the interaction of mind and language.

A different practical value of the research lies in its application for educational purposes. By applying empirical data to the established theories as well as frameworks of reference for foreign language learning and teaching, we give novel insights and recommendations on the process of teaching and learning. Although the study primarily focuses on teaching and learning Chinese, we designed the guidelines in a way that allows teachers to easily adapt them to different language combinations.

Finally, the research conclusions are not merely applicable to learning a language at the beginner's level. The results can, in fact, bring about important implications, especially in the field of translation studies, since crosslinguistic differences underlined here may arise even when we are very well acquainted with the language and culture, and yet come across unconceivable misunderstandings.

The present paper is divided into two main parts. We will briefly present the main features of our research and disclose the most pertinent empirical data in the first part, as it is important for the reader to understand the premises on which we built our research. The empirical research focuses on the thorough analysis of the interaction between the grammatical and lexical aspect for each of the three languages and how this interaction and possible systemic differences affect the way speakers of these languages verbalise particular 
aspects of their subjective reality. For this purpose, we examined the established aspectual classifications of verbs as proposed by Vendler (1967), Smith (1997), Xiao and McEnery (2004), Peck, Lin, and Sun (2013), and Koenig and Chief (2008) and slightly adapted them for the needs of our research.

In the second part, we will present the guidelines and recommendations as well as part of the descriptors' draft for cross-linguistic competences (for Chinese language) that we propose as an addition to the already established language frameworks of reference for foreign languages.

But first, let us introduce some theoretical considerations on the influence of language on the way we think. Our main concern throughout the whole study was the intricate relation between language, culture and cognition. By ploughing into the various theoretical positions on the relation between language and mind, we tried to answer the following questions: Whether and how does language affect the thought and behaviour of individuals? The analysis of diverse and sometimes even opposing scientific discourses that investigate these questions, made possible the conception of very constructive inferences, regarding not only linguistic but also social and cultural as well as cognitive aspects of Chinese, Slovenian and Italian language, for instance, the comparison between synthetic and analytic thought.

The entire study can be identified by four main research objectives that have empirically led us towards the application of results.

The first objective derives from the theoretical hypotheses of linguistic universalism, but primarily on linguistic relativity. In fact, our vision of the cognitive role of language is based primarily on the notions put forward by Lucy $(1997 ; 2005)$ rather than the controversial theory of Wharf-Sapir, therefore a more contemporary and agreeable version of this theory.

Moreover, we conceived our empirical study on the comparison of differences instead of similarities between languages that can cause the emergence of negative transfer in the process of acquiring a new foreign language. Our study encompasses the interaction between language and cognition, but also between language and culture, in the way Agar $(1991 ; 1994)$ formulates his notions of languaculture and rich points. Interestingly, when analysing the frameworks of reference discussed in the second part of this paper, we found that focusing on the differences between language systems for language learning and teaching appears less present than focusing on the similarities. We assume this derives from a more general cognitive principle of men establishing common patterns by looking for analogies. (Holme 2010)

The third objective of the study was to focus on the analysis of verbal ac- 
tions, specifically, on how lexical and grammatical aspects are verbalised in the three studied languages and how they reflect the respective models of conceptualisation, assuming that verbs help understand the way the world that surrounds us has been conceived.

The fourth objective, which is also the most pertinent to the present paper, was the application of the acquired results and conclusions to the field of foreign language learning and teaching, focusing on learning and teaching Chinese as a foreign language. We attempted to answer the question: How does the verbalisation of reality differ between our native and a foreign language? We found this question relevant, as we believe that speaking a foreign language does not necessarily mean thinking in that language. This is even more true when we begin to learn a new language and we heavily lean on notions and patterns valid in our native language. (Huang et al. 2013)

The research is based on two hypotheses and two research questions. The hypotheses, partly defined in the research objectives on the polysemic nature of verbal actions and the ambiguity of their mental representations in languages, were summed up as follows: (1) verbal actions and their mental representations are polysemic and therefore difficult to compare between languages, and (2) the nature of the Chinese verb in its most basic monomorphemic form enables a broad interpretive freedom of the degree of completion, i.e. is more focused on the process than on the result of the action performed. Based on our knowledge of the aspectual systems and how they function in the three languages, we assumed the Chinese verb emphasises more the processual phase of the action than the same verb in Italian and Slovenian, causing their speakers to respectively transfer this emphasis in the process of verbalisation as well as in conceiving reality.

The conclusions acquired with the investigated hypotheses were then applied to the research questions to design guidelines and recommendations for the development of cross-linguistic awareness, not only for learning and teaching Chinese as a foreign language, but for foreign language learning in general. The questions asked were (1) how do we define morpho-syntactical critical points ${ }^{1}$ for foreign languages? and (2) which approaches and techniques in foreign language learning and teaching can help us include contents for developing cross-linguistic awareness in a most structural and comprehensive manner.

${ }^{1}$ Critical points are those most complex morpho-syntactic and semantic points of variation between languages that can lead to negative transfer when learning a language and are a source of frustration in the process of foreign language acquisition. 
Following the first objective of this research, we critically analysed the arguments in favour and against linguistic universalism and relativism, especially what previous studies in the fields of philosophy, psychology, contrastive analysis, language anthropology, and cognitive grammar have to say about the role of language, culture and cognition affecting our perception of reality. Although they use different research questions, techniques, and methodologies, most of them agree that discrepancies in grammatical structures and the intrinsic semantic nature of the verbal action between languages can ontologically affect the way people of different language backgrounds conceive and understand reality. As sinologist and linguist James H.-Y. Tai put it: 'Each individual language thus represents a unique segmentation of the external world and the universe of human experience' (Tai 2013, 60).

\section{Research Category}

We chose verb as the main category to investigate how language affects our perception of reality, as in our opinion it is one of the most fundamental language categories and its verbalisation appears essential for understanding the creation and conception of meanings and behaviours as ongoing processes in the person's mind. Also, we identify this category as one of the more semantically abstract categories, which is why mastering semantic discrepancies between the target and native language represents one of the biggest challenges for foreign language learning. We empirically investigated the verbal aspect, lexical as well as grammatical, with specific focus on the different degrees of attention given by each language to the process on one hand and to the completion of the activity on the other.

\section{Verb Classification}

In many languages, the verb is inflected and encodes tense, aspect, mood and voice. It often also helps convey person, gender and number of the subject or object. Nevertheless, not all of the languages in the world present these features. It has been previously proposed that different languages take different perspectives on activities and events. Scholars like Ikegami (1985) have worked on the difference between Japanese and English, arguing that Japanese is a process-oriented and English is a result-oriented language. Basically, the perspective on how we understand an action being focused on the process, which might or might not end up in a change of state or towards a result made possible by this change, depends on how this action is expressed through the use of the verb. Nevertheless, not only the verb can be 
the carrier of this perspective. Language is full of more or less subtle mechanisms, which even subconsciously convey what is the conveyer's standpoint or what segment of the action he or she is focusing on.

Verbs in the Indo-European linguistic tradition have been, following Vendler's classification (1967), divided into four main types according to their inherent property of (semantic) eventuality; verbs which express state, activity, achievement, accomplishment and semelfactive as a separate category, subsequently added to Vendler's classification by Bernard Comrie in 1976. Let us have a look at how these categories work in the three languages investigated in our research. For Chinese, this division seems not to fit entirely, especially in terms of the categories of achievements and accomplishments. When we try to classify verbs in Slovenian, we are dealing with a great interconnectivity between verb class and aspectual pairs, which makes it difficult to directly apply Vendler's classification. Slovenian presents a pretty elaborate system made complex by the grammatical aspect of the verb, for which every verb has two forms, the perfective (dovršnik) and the imperfective (nedovršnik). To translate Vendler's test phrases used to classify verbs (in English) to Slovenian, we would need to switch from perfective to imperfective form and vice versa. Even though Slovenian and Italian know the same categories of lexical aspect, they do not overlap entirely, since Italian does not express grammatical aspect through single lexemes like Slovenian, but uses the past tense to emphasise the completion of an action. In Chinese, a single verb is very often not enough to express completion of an action. In these cases, the Chinese language employs resultative and compound causative constructions, which are, simply put, either a combination of two verbs or a verb and an adjective, where the first one conveys the activity and the second one the realisation that transfers the focus from the activity in process to its result. We can find more evidence of the lack of real accomplishment verbs in Chinese in Zhao (2005). Besides the resultative and compound causative construction, a maybe even more common way of changing aspect in Chinese is by employing the aspectual marker 'le.' But since it sometimes also affects only the tense, its reliability in this concern is, so to speak, weak. What can be deduced from previous studies on the ambiguities and peculiarities in the interpretation of the expressed completion of some Chinese verbs (Koenig and Chief 2008; Peck, Lin, and Sun 2013; Thepkanjana and Uehara 2009; 2010) is that they comprise a very wide and ambiguous scale of degree of completion, which is often open to interpretations.

The analysis of language tests employed in the empirical study required a categorisation of verbal actions able to comprise as much as possible the fea- 
Table 1 Proposed Features of Verb Classes and Their Categorisation

\begin{tabular}{lllllll}
\hline Verb type & {$[ \pm$ dynamic] $[ \pm$ scalar $]$} & {$[ \pm$ bounded $][ \pm$ telic $]$} & {$[ \pm$ result $]$} & Example \\
\hline Activity & + & - & - & - & - & Play \\
Semelfact./iter & + & - & \pm & - & - & Sneeze \\
semelfactive & & & & & & \\
Accomplishment & + & + & + & + & - & Build \\
Achievement & + & + & + & + & + & Find \\
\hline
\end{tabular}

tures of all three languages and at the same time preserve as high as possible the degree of universality to keep the features abstract while interpreting the results. We designed our categorisation of lexical aspect on the basis of those already established, i.e. the one from Smith (1997), Xiao and McEnery (2004), Peck, Lin, and Sun (2013) and Koeing and Chief (2008). While all of these categorisations contributed a great deal to ours, they did not entirely fit the language combinations of our choice. Hence the need for a new adjusted categorisation, which in our opinion best comprises also the features of verbal actions in general.

\section{Methods and Techniques}

A descriptive and causal nonexperimental method was applied in the study, which used research instruments to assess the subject's perception about the level of completion of the action expressed by verbs. The investigation used a within-subject design, thus each of the subjects participated in all conditions. We collected quantitative data with an off-line technique. We performed five tasks in the form of language tests. We employed different techniques to collect the data, i.e. the rating scale technique that comprised the semantic differential method, triads sorting task, in which participants had to select an option according to the associative principle, importance assessment and sorting task, free text description and arbitrary sentence formation. All of them have been designed to assess the focus on action (process or result), and how it is perceived by native speakers of Chinese, Italian and Slovenian. The sample size was $30(n=30)$, for which we tried to make groups for each language as balanced as possible in regard to gender and study field. The ages of the subjects involved in the tests ranged between 19 and 29 years, all of them were undergraduate or postgraduate students.

\section{Procedure}

The instruments employed in three tasks were questionnaires built and performed with the open-source application PsychoPy. The two tests compris- 

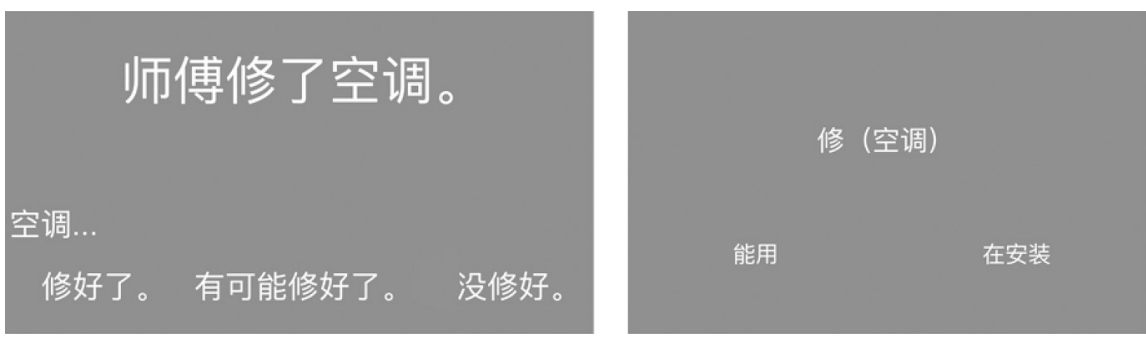

Figure 1 Screenshots of the Chinese Version of Task 1 (left) and 2 (right) Designed in PsychoPy

ing free text description and arbitrary sentence formation were designed and performed using Word document.

The main objective of Task 1 was to determine the degree of completion of verbs perceived by native speakers. The participant described his/her perception of the action expressed by the verb used in the sentence on a descriptive 3-point rating scale, stretching from not completed, maybe completed to completed. The stimulus in this task, represented by a whole sentence in which a monomorphemic verb is used along with the particle 'le' in Chinese, is expected to make participants more likely to opt for the highest degree of completion. For the Italian version of this task, the same sentences were formed with the verb in the past tense Passato prossimo. This task was not repeated in Slovenian since the selection of the verb form (dovršnik or nedovršnik) would unambiguously affect the subjects' response. In Task 2, the participants were given a verb as a stimulus, which they then had to pair with one of the two possible options (another verb or a short phrase), on the basis of semantic similarity. The two options were semantic extremes in the degree of completion, and semantically related to the stimulus. In both tasks, verb corpus comprised 76 monomorphemic verbs, (32 accomplishment verbs, 31 achievement verbs, 8 activity verbs and 5 semelfactives). Activity and semelfactive verbs were also included in the tasks in the role of distractors.

In Task 3, subjects had to asses 8 short stories presented as cartoon strips (series of drawings) for which each drawing represented a specific phase of the action and corresponded to a specific activity also described by an appropriate verb. The assessment of verbs had to be made according to the importance they ascribe to the activity in relation to the development of the story. In total, they were assessing 37 verbs; 17 of which were atelic and 20 telic.

Task 4 simply comprised of two drawings of a specific situation for which 
Figure 2

Screenshot of Task 3 (Italian Version)

(1) camminare

(2) vedere

(3) sporgersi

(4) cadere

(5) annegare

(6) tirare fuori

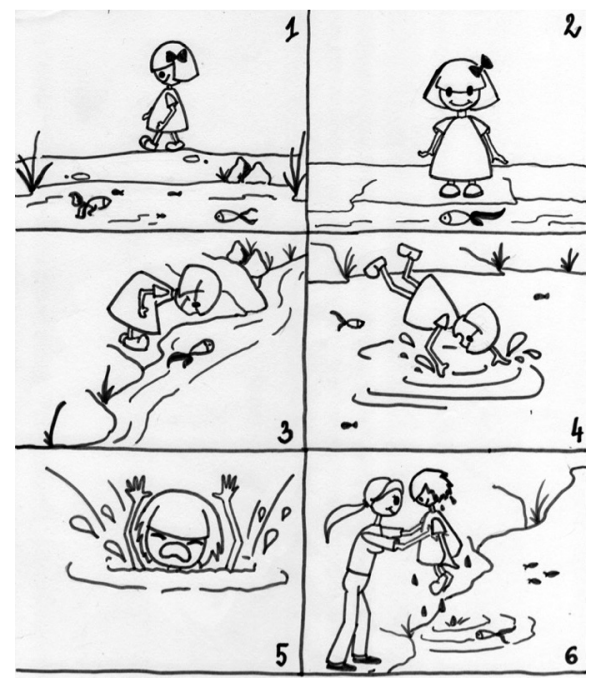

we asked the subjects to describe the illustrated situation in their own words.

In Task 5, subjects were given 16 verbs with which they had to form arbitrary sentences. The verb list comprised 5 achievement verbs, 5 accomplishment verbs, 3 activity verbs and 3 semelfactives. For Italian, the verbs were given in their infinitive form, for Slovenian we chose the infinitive of the imperfective.

\section{Empirical Evidence and Conclusions}

On the basis of the collected and analysed data, we can conclude that verbs are very polysemous in their nature, which produces semantic discrepancies between languages. By this, we can also confirm our first hypothesis that verbal actions and their mental representations are polysemous and therefore difficult to compare between languages. Which leads us to our first subhypothesis, according to which the interdependency between verb class and the degree of completion of an action in Chinese will show a smaller correspondence that the one in Slovenian and Italian, which are languages that express the verbal aspect more straightforwardly. According to our data, we can confirm that the verb class affects meaning and therefore the expressed degree of completion in all three languages. Especially Task 1 and 2 showed that verb class and degree of completion are much more interdependent in Italian than in Chinese. We also determined that it is precisely in Chinese that the category boundary between accomplishment and activity verbs is the most blurred, which can be understood as if accomplishment verbs in Chi- 
nese, reflect less telic potentiality than the same verbs in Italian and therefore Chinese speakers perceive these verbs as atelic, meaning that they do not necessarily lead to a result. Although it was difficult to prove that the Slovenian verb displays a greater interrelation between verb class and degree of completion, it is precisely this feature that serves as evidence for the degree of completion, which is self-evident in the form of the verb. On the other hand, for Chinese and Italian, where the degree of completion of the verb is not lexically defined, we confirmed that Italian verbs are more affected by class than the Chinese ones.

The data analysis also confirmed our second hypothesis about the nature of the Chinese verb, which enables a broad interpretative freedom of degree of completion, and therefore focuses more on the processual phase than on the result of the action. Subsequently, our results revealed Italian and Slovenian being more focused on the resultative phase of the action.

The investigation and analysis of the empirical data has confirmed the role of the native language as a tool of thinking of and conveying subjective reality, namely the language is saturated with conceptual activity and language thinking plays an important role in activities of communication. We tried to apply these conclusions to language teaching and learning approaches to improve didactic practices, especially in regard to the relation between language, mind and culture.

\section{Pedagogical Applications}

We have now come to the most important part of the present paper - the application of the acquired empirical results to the field of teaching and learning foreign languages. For this, we asked ourselves two main research questions, which have helped us design guidelines and recommendations for cross-linguistic awareness development, based on the interrelation between mental processing, language, and culture. Following the theory of rich points proposed by Agar (1994) and his assertion on how understanding a language goes far beyond grammar and dictionary, we find rich points (we redefined this notion for cross-linguistic purposes and named it critical points between languages) revealing and very important for foreign language learning and teaching. For instance, our empirical data showed how Chinese verbs could represent a critical point in the process of learning Chinese as a foreign language, at least for speakers of Slovenian and Italian.

To answer our first research question, and although it is impossible to universally define morpho-syntactic critical points for a foreign language in general, as each language displays some degree of idiosyncrasy that produces 
a unique configuration of critical points in relation to another foreign language, we will try to do so by focusing on our research category - verbal action. There are some general recommendations that can help us define morpho-syntactic critical points between languages. First of all, we must have thorough knowledge of the languages in question to be able to perform a detailed morpho-syntactic analysis. Besides grammatical features, it is very important to include sociolinguistic and sociocultural features of the native speakers in the investigation. On the basis of this knowledge, we have to perform a thorough theoretical and/or empirical research for the selected category that will comprise approaches established in the fields of linguistics, cognitive linguistics and intercultural communication, very similar to the one we presented earlier.

Moreover, one of the most important conclusions in relation to the second research question (approaches and techniques applied to foreign language learning and teaching) is the current lack of cross-linguistic contents. We therefore highly recommend the inclusion of these contents that have to be designed according to approaches and techniques of cognitive linguistics, as the novelty of our research is precisely the integration of the mentioned field with cross-linguistic contents for foreign language learning and teaching.

We now continue with the introduction of our final objective, namely, the presentation of guidelines and recommendations to support learners and teachers in the process of learning and teaching Chinese as a foreign language. These are important for learners, as they will facilitate the recognition of the so-called critical points between native and foreign language and the development of cross-linguistic competences as a strategy for avoiding or mastering negative transfer. For teachers, we provide specific guidelines and teaching strategies for Chinese as a foreign language, based on approaches of cognitive linguistics, foreign language teaching methods as well as theories established in the fields of intercultural and cross-linguistic studies. We divided the proposed guidelines for developing cross-linguistic awareness competences into four sets subdivided between learning and teaching categories. The sets are as follows: (1) recommendations for teachers' language competences, (2) guidelines for learning content designers, (3) guidelines for the teaching process, (4) guidelines for the learning process.

Although the guidelines and recommendations were conceived for the purpose of learning and teaching Chinese, they were designed in a way that allows their adaptation and application for teaching and learning other foreign languages. However, they are most applicable to unrelated languages. 
To achieve our final research objective, to boost language competences, it is necessary to include more comprehensively cross-linguistic awareness competences to the field of language learning and teaching. An example taken from our research would be to include in the foreign language curriculum more thorough explanations about how verbal actions are conceived and conceptualised in one language compared to another.

Frameworks of reference for languages are valuable instruments for the applied part of our research. In fact, the analysis of such instruments, used to describe the achievements of learners of foreign language across the world, highlighted a general lack of cross-linguistic competences.

Our analysis of frameworks of reference focused on frameworks for foreign language teaching and learning used in Europe and those established in the field of learning and teaching Chinese as a foreign language around the globe. We analysed and compared the Common European Framework of Reference - CEFR (Kovačič et al. 2011) and the Companion Volume with new descriptors, the Framework of Reference for Pluralistic Approaches to Languages and Cultures - FREPA (Candelier et al. 2017), the International Curriculum for Chinese Language Education (Hanban 2009), the Chinese Language Proficiency Scales For Speakers of Other Languages (Hanban 2007), and The New HSK. ${ }^{2}$ While the CEFR, the CEFR Companion Volume and the FREPA are frameworks designed for no language in particular and can be applied for teaching and learning foreign languages in general, the Curriculum, the Scales and the HSK, on the other hand, are language-specific and are designed to fulfil the requirements of learning and teaching Chinese as a foreign language.

Our investigation firstly focused on how frameworks of reference address cross-linguistic discrepancies that are language-specific and are based on behavioural and mental patterns.

Even though the authors of the CEFR acknowledged the importance of a learner's mental context in the process of creating meaning, they conclude that how he or she recognises external circumstances and limitations, accepts them, adapts or does not adapt himself or herself to them depends on his or her personal interpretation of the situation that is affected by his or her previous knowledge, values and beliefs. They also mentioned the difficulties in overcoming differences in values and beliefs, courtesy conventions, social expectations and other aspects that affect the way learners interpret interaction if they do not develop a relevant intercultural awareness.

\footnotetext{
${ }^{2}$ The New Chinese Proficiency Test, see http://www.hanban.edu.cn/tests/node_7486.htm\#no1.
} 
Although these facts, which are mainly considering intercultural awareness, are acknowledged, they do not really show up among the competences required for developing cross-linguistic awareness.

A basic weakness of the CEFR is also its fundamental orientation towards the user (what he or she is supposed to know) compared to the focus it gives to language as a system (how it works). Another flaw we identified in the CEFR is that even though the descriptors were designed for a plurilingual user, the language competences are very singular, for one language, which makes it faulty in terms of metalinguistic and cross-linguistic competences. The integration between languages is known only in the framework of intercultural contents.

The CEFR - Companion Volume with new descriptors was created in 2018 to remedy the deficiency of CEFR from 2001. We found that the Companion Volume gives more recognition and emphasis to cross-linguistic and cognitive mediation, acknowledging their importance for a successful communication. Moreover, it also emphasises the need to precisely define the languages involved in the communication process. This addition to the CEFR, despite not being exhaustive enough, is a step forward in regard to crosslinguistic awareness competences, which confirms the aim of our research and makes it relevant for future improvements of frameworks of reference.

Like the Companion Volume, the FREPA was created to update certain aspects of the CEFR. While the CEFR was designed on more methodological grounds, the FREPA complements it in terms of the tools that focus primarily on the development of users' plurilingual and intercultural competences and knowledge through pluralistic approaches towards languages and cultures. The novelty of the FREPA, which is also the most important aspect for the present study, is the principal of awakening to languages and the mutual understanding of related languages. The FREPA is therefore much more focused on plurilingualism and the competences it demands. For instance, the FREPA emphasises 'enabling learners to have easier access to a specific language or culture by using aptitudes acquired in relation to / in another language or culture [...]'. (Candelier et al. 2017, 16). By acknowledging this, the authors of the FREPA address a fundamental principle for the present study - cross-linguistic awareness, which is very often neglected on account of intercultural awareness.

The comparison of the three descriptors shows how the FREPA is much more thorough in including competences related to cross-linguistic aspects and linguistic awareness. In some way, the pointing out of these differences, not only cultural but linguistic as well, which create diverse perceptions of 
reality, proves that notions of cognitive linguistics are already part of the institutionalised teaching methods.

The main limitation of the analysed frameworks of reference in terms of foreign language teaching practice is that they are still very general, although they thoroughly define the competences, thus making the guidelines pretty vague. Moreover, they do not indicate that the frameworks have been primarily designed for Indo-European languages, which have very similar systemic properties and can be therefore taught using similar principles.

Specifically for Chinese as a foreign language, we identified three instruments that could be compared to the European framework of reference for languages: the International Curriculum for Chinese Language Education, the Chinese Language Proficiency Scales For Speakers of Other Languages, and The New HSK (The New Chinese Proficiency Test). Interestingly, the Chinese Language Proficiency Scales were created on the basis of the CEFR and represent a standard for designing curricula for teaching Chinese as a foreign language, for writing Chinese language textbooks and for assessing language proficiency for learners of Chinese as a foreign language. The International Curriculum is a rather recent instrument for planning the teaching process and assessing language proficiency and serves as the basis for designing teaching resources. The Curriculum addresses the importance of interactions between aspects of language and culture, but vaguely points out the importance of plurilingualism and how the structure of our native language affects the learning of a foreign language and the ways we mentally conceive reality. The latter is only mentioned indirectly and without giving any evidence on which cognitive linguistics is based on. On the other hand, much more importance is given to contents related to intercultural communication and intercultural awareness.

Moreover, the Chinese Language Proficiency Scales and The New HSK do not give much emphasis on the understanding of a language system as a deeper cognitive process that can affect perception, conceptualisation and the way native speakers think and consequently generate the content of their message. This idea is mentioned only in the Curriculum.

\section{Guidelines and Recommendations for Developing Cross-Linguistic Awareness Competences}

As we pointed out several times in the previous pages, we find that the currently established frameworks of reference for languages lack contents for specific aspects, i.e. the development of cross-linguistic awareness competences, which are of great importance, especially for learning and teaching 
unrelated languages like Slovenian and Chinese. The final object of our research was to fill this gap by designing guidelines and recommendations applicable in the course of foreign language learning and teaching.

The following guidelines and recommendations for developing crosslinguistic awareness competences have been divided into four sets subdivided between learning and teaching categories.

\section{Recommendations for Teachers' Language Competences}

Cross-linguistic differences are an important aspect of foreign language learning but are usually vaguely addressed in frameworks of reference, especially in comparison to the intercultural differences; therefore, we consider teacher's competences a fundamental aspect of this process. To be able to teach cross-linguistic dimensions for Chinese as a foreign language, it is of crucial importance to have thorough knowledge of the languages used by the learners in the learning process (native and foreign language). Most Chinese teachers, who are often rated higher for their language authenticity, do not speak the native language of the learners, and are therefore not suited to teach cross-linguistic contents. We therefore recommend Sinologists or Chinese teachers who speak the native language of the learners to teach Chinese as a foreign language. The same applies to teachers of other foreign languages.

\section{Guidelines for Learning Content Designers}

To those who design learning contents, materials and resources and want to include contents for the development of cross-linguistic awareness, we propose to consider the approach that encompasses the relational contact between the self and the other. By learning the language, we also learn new ways of thinking and by doing this, the learner does not have to give up his or her own identity, but rather accept the changes that this learning introduces into his or her hitherto valid personal perspectives. We suggest following the six phases for the conceptualisation of intercultural sensitivity proposed by Bennett (1993). We have adapted his phases for the conceptualisation of cross-linguistic awareness.

\section{Guidelines for the Teaching Process}

The aforementioned phases primarily proposed to help and guide designers to generate learning contents for developing cross-linguistic awareness can, along with language competences descriptors, also represent support 
for teachers that can follow the phases in the teaching process and successfully link them to the relevant exercises. It is a teacher's responsibility to avoid critical points to discourage learners in the process, but rather make negative transfer become part of the learner's learning path.

In our study, we have designed the most important descriptors for crosslinguistic competences (which are partly based on the analysis of the frameworks of reference) available for teachers of Chinese as a foreign language to include in their curricula. Competences descriptions are mainly linked to the field of language, which according to this study is organically related to mind and culture. We would like to share in the present paper some sections of the designed descriptors, to enable the reader to better understand our basic idea of cross-linguistic competences.

The descriptors have been designed according to the phases the learner has to pass through while acquiring language sensibility and cross-linguistic awareness. The domains according to which the phases are defined are awareness, knowledge and skills. Not all domains are fundamental in all phases. For instance, awareness is very important in the first three phases where the learner has to advance from the negative to the positive stand towards a foreign language, while skills are much more crucial in the final phases. Phases progress according to the level of knowledge already established by the current frameworks of reference but they do not overlap completely for the process, and attainment of cross-linguistic awareness is specific for each learner. It is advisable for the teacher to set a scale to better assess a learner's progress towards the target. In this way, we do not assess the level of knowledge, but rather help the learner to develop awareness about his or her role in the process of learning. We would also like to remind the reader that the proposed descriptors are a draft that needs to be improved in the coming years.

\section{Guidelines for the Learning Process}

Teachers can decide to introduce qualitative methods of self-assessment in their class, for instance, the language portfolio. In this case, the previously introduced descriptors can be of great help to learners in assessing their own cross-linguistic experience and the degree of progression through the stated phases. This should undergo the assessment of the awareness of cross-linguistic differences, of the understanding of the acquired principles of cross-linguistics and the actual use within the contents of the acquired cross-linguistic knowledge.

When teachers are not using portfolios, they should encourage the de- 
Table 2 Descriptors Proposal for Developing Cross-Linguistic Awareness (Draft)

\begin{tabular}{|c|c|c|}
\hline Phase & Domain & Description of the target for progressing through phases \\
\hline $\begin{array}{l}1 \text { Differences } \\
\text { denial }\end{array}$ & Awareness & $\begin{array}{l}\text { - Perceives the existence of differences between languages, } \\
\text { which are of systemic nature. (Example: verbal aspect.) } \\
\text { - Perceives that each language has its own specific way of } \\
\text { conceiving and verbalising reality. (Example: differences be- } \\
\text { tween languages in emphasising the phase of process or } \\
\text { result of the action.) } \\
\text { - Perceives the differences between ways of thinking of } \\
\text { speakers of different languages. (Example: the comparison } \\
\text { between Chinese synthetic and the Western analytic think- } \\
\text { ing.) } \\
\text { - Perceives the specificities of linguistic communication in } \\
\text { his or her own language compared to Chinese. (Example: } \\
\text { grasping the meaning through context in Chinese.) }\end{array}$ \\
\hline $\begin{array}{l}4 \text { Differences } \\
\text { acceptance }\end{array}$ & $\begin{array}{l}\text { Knowledge, } \\
\text { skills }\end{array}$ & $\begin{array}{l}\text { - Knows that each language has its own specific way of con- } \\
\text { ceptualisation in verbalisation of reality. (Example: differ- } \\
\text { ences between languages in emphasising the phase of pro- } \\
\text { cess or result of the action.) } \\
\text { - Knows that Chinese speakers can interpret reality different- } \\
\text { ly because of specific values, cultural system, language sys- } \\
\text { tem, etc. (Example: the comparison between the holistic } \\
\text { understanding of relationships and the understanding of } \\
\text { meaning contextually.) } \\
\text { - Knows that critical points can be the cause of mistakes and } \\
\text { negative transfer from his or her native language. (Example: } \\
\text { the direct use of the aspectual marker -le for defining past } \\
\text { tense.) } \\
\text { - Can identify differences between his or her native language } \\
\text { and Chinese and verbalises it. (Example: the use of aspec- } \\
\text { tual markers in Chinese compared to the aspect in Slovene, } \\
\text { which is expressed at a lexical level.) } \\
\text { - Can investigate (autonomously or under a teacher's guid- } \\
\text { ance) operating modes of his or her native language and } \\
\text { Chinese. } \\
\text { - Can compare morpho-syntactic differences between lan- } \\
\text { guages and connects them with the semantic ones. (Ex- } \\
\text { ample: the meaning of process and the use of imperfective } \\
\text { verbal aspect.) }\end{array}$ \\
\hline
\end{tabular}

Notes For the whole descriptors proposal see Čok (2019).

velopment of cross-linguistic competences by guiding learners with special exercises and activities. Learners have to test themselves in conceptualising meanings in the most authentic manner, which can only be achieved when he or she is aware of the differences between ways of conceptualisation established in his or her native and foreign language. 
As a concluding remark, we would like to propose one final recommendation for designing curricula that consider and raise awareness of crosslinguistic aspects. It is very important that we thoroughly introduce and explain language-related contents in the initial learning phases, as the learner is facing an enormous quantity of unknown information, especially at the beginning. The designer of the curriculum will therefore have a lot of work with finding correlations between language structures and their manifestation in a more general conceptual reality of speakers of the foreign language. Our study, which can serve as an example, was mainly concerned with verbal aspect, while some noun features are also vaguely mentioned. When designing actual learning activities, the teacher should aim at encouraging a broader understanding of the structure's meaning and the relation between linguistic (morpho syntactic, semantic, etc.) and conceptual categories.

\section{References}

Agar, Michael. 1991. 'The Biculture in Bilingual.' Language in Society 20 (2): 16781.

-1994. Language Shock: Understanding the Culture of Conversation. New York: William Morrow.

Bennett, Milton. J. 1993. 'Cultural Marginality: Identity Issues in Intercultural Training.' In Education for Intercultural Experience, edited by Michael Paige, 109-35. Yarmouth, MA: Intercultural Press.

Candelier, Michel, Antoinette Camilleri-Grima, Veroniqe Castellotti, Jean-Francois De Pietro, Ildiko Lorincz, Franz-Joseph MEisner, Artur Noguerol, and Anna Schröder-Sura. 2017. Referenčni okvirza pluralistične pristope kjezikom in kulturam: zmožnosti in viri. Ljubljana: Zavod Republike Slovenije za šolstvo.

Comrie, Bernard. 1976. Aspect: An Introduction to the Study of Verbal Aspect and Related Problems. Cambridge: Cambridge University Press.

Čok, Tina. 2019. 'Konceptualizacija in upovedovanje glagolskega dejanja v slovenščini, kitajščini in italijanščini.' PhD Dissertation, Univerza na Primorskem.

Hanban. 2007. Guoji hanyu nengli biaozhun. Beijing: Waiyu jiaoxue yu yanjiu chubanshe.

- 2009. Guoji hanyu jiaoxue tongyong kecheng dagang: Yuenanyu, Hanyu duizhao. Beijing: Waiyu jiaoxue yu yanjiu chubanshe.

Holme, Randal. 2010. Cognitive Linguistics and Language Teaching. Beijing: Foreign Language Teaching and Research Press.

Huang, Jui-Ting, Junyu Li, Dong Yu, Li Deng, and Yifan Gong. 2013. 'CrossLanguage Knowledge Transfer Using Multilingual Deep Neural Network with Shared Hidden Layers.' In Proceedings of the 2013 IEEE International 
Conference on Acoustics, Speech and Signal Processing, 7304-8. Vancouver: IEEE.

Ikegami, Yoshihiko. 1985. 'Activity - Accomplishment - Achievement: A Language That Can't Say "I Burned It, But It Didn't Burn" and One that Can.' In Linguistics and Philosophy: Essays in honour of Rulon S. Wells, edited by Adam Mikkai and Alan K. Melby, 265-304. Amsterdam: John Benjamins.

Koenig, Jean-Pierre, and Lian-Cheng Chief. 2008. 'Scalarity and State-Changes in Mandarin (and Other Languages).' Empirical Issues in Syntax and Semantics 7:241-62.

Kovačič, Irena, Ina Ferbežar, Simona Kranjc, Karmen Pižorn, Janez Skela, Marko Stabej, and Nataša Detič. 2011. Skupni evropski jezikovni okvir: učenje, poučevanje, ocenjevanje. Ljubljana: Ministrstvo Republike Slovenije za šolstvo in sport.

Lucy, John, A. 1997. 'Linguistic Relativity.' Annual Review of Anthropology 26:291312.

- 2005. 'Through the Window of Language: Assessing the Influence of Language Diversity on Thought.' Theoria 54: 299-309.

Peck, Jeeyoung, Jingxia Lin, and Chaofen Sun. 2013. 'Aspectual Classification of Mandarin Chinese Verbs: A Perspective of Scale Structure.' Language and Linguistics 14 (4): 663-700.

Smith, Carlota, S. 1997. The Parameter of Aspect. 2nd ed. Dordrecht: Kluwer.

Tai, James H.-Y. 2013. 'Reflections on Typological Characterization of Chinese Gramma: Human Language Resources and Linguistic Typology.' In Papers from the Fourth International Conference on Sinology, 60-88. Taipei: Academia Sinica.

Thepkanjana, Kingkarn, and Satshi Uehara. 2009. 'Resultative Constructions with "Implied-Result" and "Entailed-Result" Verbs in Thai and English: A Contrastive Study.' Linguistics 47 (3): 589-618.

- 2010. 'Syntactic and Semantic Discrepancies among the Verbs for "kill" in English, Chinese and Thai.' In PACLIC 24 Proceedings, 291-300. Tokyo: Waseda University.

Vendler, Zeno. 1967. Linguistics in Philosophy. Ithaca, NY: Cornell University Press.

Xiao, Richard, and Tony McEnery. 2004. Aspect in Mandarine Chinese: A CorpusBased Study. Amsterdam: John Benjamins.

Zhao, Yang. 2005. 'Causativity in Chinese and Its Representations in English, Japanese and Korean Speakers' L2 Chinese Grammars.' PhD dissertation, University of Cambridge. 